\title{
Quantitative Analysis of the Flavonoid Mesquitol in the Medicinal Plant Prosopis juliflora with Seasonal Variations in Marigat, Baringo County-Kenya
}

\author{
Mark Peter Odero ${ }^{1,}$, Were Lincoln Munyendo ${ }^{2}$, Ambrose Kipchumba Kiprop ${ }^{1}$ \\ ${ }^{1}$ Department of Chemistry \& Biochemistry, Moi University, Eldoret, Kenya \\ ${ }^{2}$ School of Pharmacy \& Health Sciences, United States International University - Africa, Nairobi, Kenya
}

Email address:

miakitah@yahoo.com (M. P. Odero), lmunyendo@usiu.ac.ke (W. L. Munyendo), ambkiprop@gmail.com (A. K. Kiprop)

*Corresponding author

\section{To cite this article:}

Mark Peter Odero, Were Lincoln Munyendo, Ambrose Kipchumba Kiprop. Quantitative Analysis of the Flavonoid Mesquitol in the Medicinal Plant Prosopis juliflora With Seasonal Variations in Marigat, Baringo County-Kenya. Science Journal of Analytical Chemistry.

Vol. 5, No. 6, 2017, pp. 107-112. doi: 10.11648/j.sjac.20170506.16

Received: November 7, 2017; Accepted: November 20, 2017; Published: January 2, 2018

\begin{abstract}
Several studies indicate that mesquitol exhibits significant free-radical scavenging properties, antioxidant and $\alpha$-glucosidase inhibitory properties. This makes it a useful resource with potential for exploitation in both agro-food and pharmaceutical industries. The heartwood of the plant $P$. juliflora is noted to contain very high levels of the flavan-3-ol compound mesquitol. Previous studies showed that in non-tropical countries like the United States of America where they experience four seasons in a year, mesquitol shows varying seasonal abundance with the least abundant season being the winter period. Meanwhile limited data exists on its abundance trends in tropical arid and semiarid regions that only experience the dry and wet seasons in a year. Plants samples of two distinct age groups were collected during the dry and wet seasons and extracted serially via a soxhlet apparatus after which column chromatography was used to assist in the process of mesquitol isolation. Thin layer chromatography assisted in pooling of fractions with similar $\mathrm{R}_{\mathrm{f}}$ values. The $\mathrm{R}_{\mathrm{f}}$ values were compared to available literature to ascertain fractions that contained mesquitol. A HPLC-UV method was developed and validated illustrating high accuracy and precision for the quantification of the mesquitol content in different $P$. juliflora samples. On evaluation, mesquitol was found to be more abundant during the wet seasons reaching $642.893 \mu \mathrm{g} / \mathrm{ml}$ as compared to $181.245 \mu \mathrm{g} / \mathrm{ml}$ for dry seasons. This abundance could be attributed to chemo-seasonal dynamics that have been witnessed to affect biosynthesis and deposition of phyto-compounds in plants. The developed and validated HPLC method, illustrated satisfactory quantification of mesquitol at $3-6 \%$ of the crude extract. This paper presents useful information on seasonal abundance dynamics of mesquitol in the tropics as a basis for the resource valorization.
\end{abstract}

Keywords: Seasonal Dynamics, Mesquitol Abundancies, Mesquitol Quantification

\section{Introduction}

Secondary metabolites are a group of compounds that are of low molecular weight and that are naturally present in a plant in significant quantities alongside hemicellulose, lignin and cellulose [1]. The compound mesquitol, is a flavan-3-ol compound, chemically known as (2R, 3S)-2-(3, 4-dihydroxyphenyl)-3, 4-dihydro-2H-chromene-3, 7, 8-triol (Figure 1). It is an antioxidant with $\alpha$-glucosidase inhibitory properties and radical scavenging properties. It has been illustrated to stimulate the endogenous body defence system hence capable of treating and preventing diseases like cancers and diabetes $[2,3]$. In fact, studies done on the antioxidant capabilities of mesquitol, show that when compared to two major antioxidants currently marketed as medicines i. e. probucol and $\alpha$-tocopherol, it was found to be a stronger antioxidant than both. This showing its great potential as an active pharmaceutical ingredient $[4,5]$.

Presently, there is a shift from synthetic to traditional forms of medicine being witnessed world over. This is due to the 
high toxicity and undesirable long term side effects of most of the synthetic drugs used in the market hence the potential importance and applicability of mesquitol [6]. Apart from this it has also been suggested that mesquitol has great potential for use as a lead natural organic compound that can be used for future development of anti-glycating agents with potent antioxidant properties [7]

It has been noted that despite the existence of gene expression, genetic control and genotypes, the amount of plant extractives found within a plant greatly vary as a result of seasonal and climatic variables including, availability of water, temperature, UV light, altitude, availability of nutrients, environment, the stage of growth and age [8,9]. This is because the process of the production of plant metabolites, is largely as a result of the adaptive process of the plant in response to external factors surrounding it. It is for this reason that plant metabolites are never the same even within the same species as they will vary over time, seasons, and even geographical locations [10].

Several studies published in reputable scientific journals, indicate that there exists no general rule as to when it is best to harvest plant extractives as they vary from one plant species to another. For example, it has been noted that in some plant species, the summer period is the best to harvest phytochemicals as they are in plenty with the yields, drastically decreasing as you approach the winter period. While in some other species, it is vice versa i. e. the yield drastically increases as you tend towards winter [11]. It is therefore of great importance to therefore first study the amount of phytochemicals present in a season before delving to exploit them commercially for maximum profits and reduction of wastage [12].

Previous studies on the abundance dynamics of non-carbohydrate organic compounds of $P$. juliflora over a period of two years in the United States of America showed that non carbohydrate organic compounds were most plenty during the late spring and early summer seasons and the least during the winter season signifying great seasonal variation [13]. Whereas this data exist for places with all the four seasons, as above, there exist limited data on the abundance dynamics of the same in tropical arid and semi arid regions. The figure below shows the structure of mesquitol.<smiles>Oc1ccc([C@@H]2Oc3c(ccc(O)c3O)C[C@H]2O)cc1O</smiles>

Figure 1. Structure of mesquitol.

\section{Materials and Methods}

\subsection{Chemicals and Reagents}

Acetonitrile, water, formic acid were all of analytical grade, manufactured by Sigma Aldrich Company and supplied by
Kobian Laboratory supplies Nairobi Kenya. Pure mesquitol was sourced from LERMAB laboratories at the University of Lorraine in Nancy France. Methanol, acetone, hexane, dichloromethane were bought from Gelsap laboratories Nairobi and were of the analar grade.

\subsection{Instrument and Apparatuses}

HPLC (Shimadzu LC-10AT Liquid Chromatography system with SPD-10A UV-Vis detector) and UV- 1800 Shimadzu.

The various equipment that were used to carry out experimental procedures included blender (ramtons), analytical balance, water bath, rotary evaporator (BÜCHI Rota vapor R-114), Test tubes, Capillary tube, $50 \mathrm{~mL}$ burette, $1 \mathrm{~mL}$ pipette, $10 \mathrm{~mL}$ dropping pipette, electric oven, $250 \mathrm{~mL}$ conical flask, thin Layer Chromatography plates (Merck Company).

\subsection{Sample Collection and Processing}

The heartwood of $P$. juliflora was harvested from Marigat Baringo county (latitude $0^{\circ}, 20^{\prime} \mathrm{N}$, longitude $35^{\circ}, 57^{\prime} \mathrm{E}$ ) in Kenya. The plant samples were sorted based on the seasons of harvest as wet season and dry season and grouped according to ages as below 4 years and above 4 years.

Serial extraction was done with hexane, DCM, acetone and methanol as the extractants by taking $15 \mathrm{~g}$ of the dry ground powder of the $P$. juliflora heartwood and extracting it with 250 $\mathrm{ml}$ of the solvents via soxhlet method of extraction. The extracts were then rota-evaporated under vacuum before transferring to sets of pre weighed bottles and placed on open air to dry.

\subsection{Mesquitol HPLC Quantification}

\subsubsection{HPLC Method Development and Validation}

HPLC method was developed and validated according to ICH guidelines 2003 [14]. The HPLC analysis was performed on a Shimadzu LC-10AT Liquid Chromatography system with SPD-10A UV-Vis detector. A $\mathrm{C}_{18}(15 \mathrm{~cm} \mathrm{X} 2.1 \mathrm{~mm} \mathrm{X}$ $2.7 \mu \mathrm{m})$ luna ${ }^{\mathrm{R}}$ column was chosen for separations at a constant column temperature of $25^{\circ} \mathrm{C}$ and $\mathrm{UV}$ detection at $\lambda$ max set to $280 \mathrm{~nm}$.

The mobile phase, comprised of a mixture of acetonitrile: water (formic acid $0.1 \%$ ) in the ratios of 70:30 respectively. A flow rate of $0.4 \mathrm{mLmin}^{-1}$ and pump pressures from 8.0 MPa to a maximum of $15.0 \mathrm{MPa}$ for sustained stable flow. The auto-sampler delivered a $10 \mu \mathrm{m}$ sample to the column. The precision was established by using the Relative Standard Deviation (RSD) and the accuracy by determining the analytical recoveries intraday and interday.

Limits of Detection (LoD) and Limits of Quantification (LoQ) were calculated by simultaneous analysis of mesquitol using standard deviations of predetermined analytical recoveries.

The methods robustness was determined by purposefully altering the experimental conditions. The flowrate was adjusted slowly from $7.5 \mathrm{~mL} \mathrm{~min}^{-1}$ to $0.1 \mathrm{~mL} \mathrm{~min}^{-1}$ with the 
intention of studying the effect of flowrate on the peak resolution. The temperature was also adjusted from $25^{\circ} \mathrm{C}$ to $45^{\circ} \mathrm{C}$ then to $65^{\circ} \mathrm{C}$. To establish the linearity and range, standard solutions were diluted from stock solutions in the ranges of $10,20,40,80$ and $160 \mu \mathrm{g} / \mathrm{l}$. The correlation co-efficient was obtained from the plot of the peak area against the concentrations.

\subsubsection{Mesquitol Isolation from Extracts}

$75 \mathrm{mg}$ of each of the chosen extracts were subjected to column chromatography by eluting the samples with $10 \mathrm{mLs}$ of the reagents in the ratios of Hexane DCM (9:1) then 8:2 systematically changing the ratios of the reagents up to $1: 9$ each time collecting the eluates in approximately $10 \mathrm{mLs}$ flasks. The sample was then eluted further with DCM and Ethyl acetate also in the ratios of 9:1 systematically till 1:9 and finally ethyl acetate to acetone also from 9:1 up to 1:9. Thin Layer Chromatography (TLC) was then used to pool together samples based on $\mathrm{Rf}$ values

\subsubsection{Mesquitol Quantification}

The developed and validated HPLC method was then used to quantify the amounts of mesquitol in crude extracts for the samples this was done in the following manner. Fresh crude extracts to be used for analysis were prepared by first reconstituting a stock solution of $1 \mathrm{~mL}$ of crude extract in $100 \mathrm{~mL}$ of mobile phase that consisted of acetonitrile: water (formic acid $0.1 \%$ ) in the ratio $70: 30 \mathrm{v} / \mathrm{v}$.

Different dilutions were prepared by diluting $6.25 \mathrm{~mL}$, $12.5 \mathrm{~mL}, 25 \mathrm{~mL}$ and $50 \mathrm{~mL}$ of this stock solution in $100 \mathrm{ml}$ volumetric flask using the mobile phase to obtain samples for HPLC analysis. A volume $20 \mu \mathrm{L}$ of sample was then injected into the sample injection loop and the sample loaded into the $\mathrm{C}_{18} 15 \mathrm{~cm}$ X $2.1 \mathrm{~mm}$ X $2.7 \mu \mathrm{m}$ lunar column.

\subsection{Evaluation of Seasonal Variations}

Seasonal aspects including rainfall, humidity and temperature were evaluated as determinants of phytocompounds deposition in plants. This was done by establishing the weather patterns of Baringo County Kenya from data collected over time by the meteorological department within the plant habitat.

Archival weather data on rainfall, relative humidity and temperature were collected from the Katimok Forest Meteorological Station in Baringo county for a period of two years from January 2015 - December 2016. Daily data were aggregated into monthly and further into yearly data to established trends in seasonal aspects over this period of time.

\section{Results and Discussions}

\subsection{Mesquitol Extraction and Determination of Total Flavonoid Content}

After serial extraction had been done, with Hexane, DCM, acetone and methanol as previously discussed, they were collected in pre weighed bottles and their average crude extracts results were tabulated as in the table below. Table 1 showing the percentage crude extracts of $P$. juliflora plant.

Table 1. Percentage crude extracts.

\begin{tabular}{llll}
\hline Sample & Solvent system & Crude extract & $\begin{array}{l}\text { Percentage yield } \\
\text { Soxhlet }\end{array}$ \\
\hline WB1 & Hexane & WB1-Hex & $0.86 \pm 0.21$ \\
& Dichloromethane & WBl-DCM & $1.32 \pm 0.34$ \\
& Acetone & WBl-Ace & $3.15 \pm 0.86$ \\
& Methanol & WBl-Met & $4.21 \pm 0.81$ \\
WAb & Hexane & WAb-Hex & $2.34 \pm 0.54$ \\
& Dichloromethane & WAb-DCM & $3.68 \pm 0.51$ \\
& Acetone & WAb-Ace & $7.69 \pm 1.56$ \\
& Methanol & WAb-Met & $10.22 \pm 2.03$ \\
DB1 & Hexane & DBl-Hex & $0.84 \pm 0.27$ \\
& Dichloromethane & DB1-DCM & $0.95 \pm 0.41$ \\
& Acetone & DBl-Ace & $2.98 \pm 0.49$ \\
& Methanol & DB1-Met & $3.16 \pm 0.75$ \\
\hline \multirow{2}{*}{ DAb } & Hexane & DAb-Hex & $2.14 \pm 0.52$ \\
& Dichloromethane & DAb-DCM & $3.46 \pm 0.75$ \\
& Acetone & DAb-Ace & $7.19 \pm 1.01$ \\
& Methanol & DAb-Met & $9.86 \pm 1.21$ \\
\hline
\end{tabular}

Key: Hex: Hexane, Ace: Acetone, Met- Methanol DCM: Dicloromethane.

Subsequently, the total flavonoid content of different extracts from hexane, dichloromethane, acetone, methanol was done by the employment of the Aluminium Chloride colorimetric method as described by Hossain [15] with little modifications. This was done by taking 0.25 grams each of the extracts and dissolving them with $1.25 \mathrm{~mL}$ of distilled water and approximately $75 \mu \mathrm{L}$ and of $6 \%$ of $\mathrm{NaNO}_{3}$ added then solution thoroughly mixed.

The mixture was then incubated in a dark cabinet for approximately 6 minutes then $10 \% \mathrm{AlCl}_{3}(150 \mu \mathrm{L})$ added to each of the test tubes and subsequently incubated again for approximately 5 minutes in a dark cabinet. A standard calibration curve was obtained by using quercetin of concentrations $0,2.5,10,20,40,80 \mu \mathrm{g} / \mathrm{mL}$ diluted in methanol. This was then used for measuring of absorbance at a wavelength of $\lambda \max 510 \mathrm{~nm}$ using a UV-Vis spectrophotometer. This were done in triplicates and subsequent results averaged. The total flavonoid content was calculated using formula.

$$
\mathrm{TFC}=(\mathrm{AxB}) /(\mathrm{CxD})
$$

Where $\mathrm{A}=$ Absorbance of crude extracts

$\mathrm{B}=$ Mass of quercetin in $\mathrm{mg}$

$\mathrm{C}=$ Absorbance of standard

$\mathrm{D}=$ mass of extracts in $\mathrm{mg}$

The following calibration curve was used for the quantifying total flavonoid content in the plant extracts. Figure 2 shows the standard curve of absorption against quercetin concentration. 


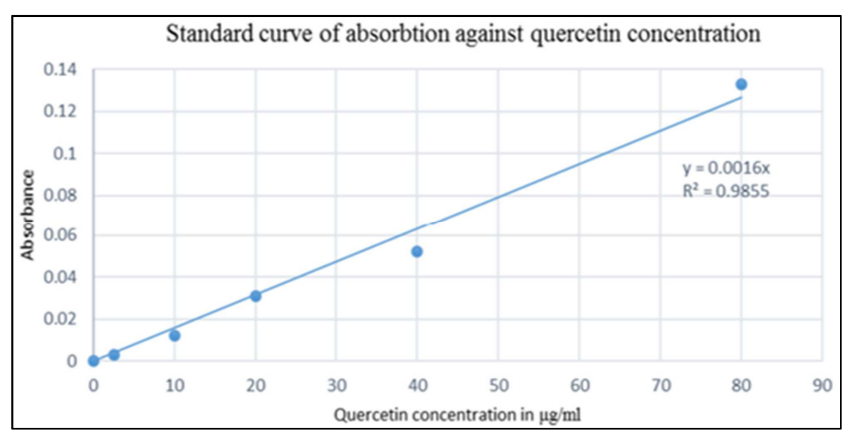

Figure 2. Shows the standard curve of absorption against quercetin concentration.

The total flavonoid contents (in mg of quercetin equivalent per gram dry weight) of the heartwood of $P$. juliflora for all crude extracts obtained with pure solvents and shown in the table 2 below.

Table 2. The total flavonoid contents (in $\mathrm{mg}$ of quercetin equivalent per gram dry weight.

\begin{tabular}{ll}
\hline SAMPLE & TOTAL FLAVANOIDS (Mg/g) \\
\hline WAb- Hex & $2.2 \pm 0.86$ \\
WAb- DCM & $5.5 \pm 1.21$ \\
WAb-Ace & $21.7 \pm 4.93$ \\
WAb- Met & $11.5 \pm 2.64$ \\
\hline
\end{tabular}

The highest amounts of flavonoids were found in the acetone extract (WAb-Ace) at $21.7 \mathrm{mg} / \mathrm{g}$ of dry weight while the lowest were recorded in the hexane extract (WAb-Hex) that afforded $2.2 \mathrm{mg} / \mathrm{g}$. This finding informed the use of the crude extract, WAb-Ace, in subsequent investigations of flavonoid mesquitol abundance dynamics in the plant $P$. juliflora and for the analytical method development

\subsection{Mesquitol HPLC Quantification}

\subsubsection{Validation of the HPLC Method}

A chromatographic method was developed, with a column temperature, of $25^{\circ} \mathrm{C}$ and the UV detector $\left(\lambda_{\text {max }}\right)$ set at $280 \mathrm{~nm}$.
The mobile phase, of acetonitrile: water (formic acid $0.1 \%$ ) in the ratios of 70:30 respectively. An optimum flow rate was established at $0.4 \mathrm{mLmin}^{-1}$, with a retention time for mesquitol being 3.5 minutes (figure 3 ).

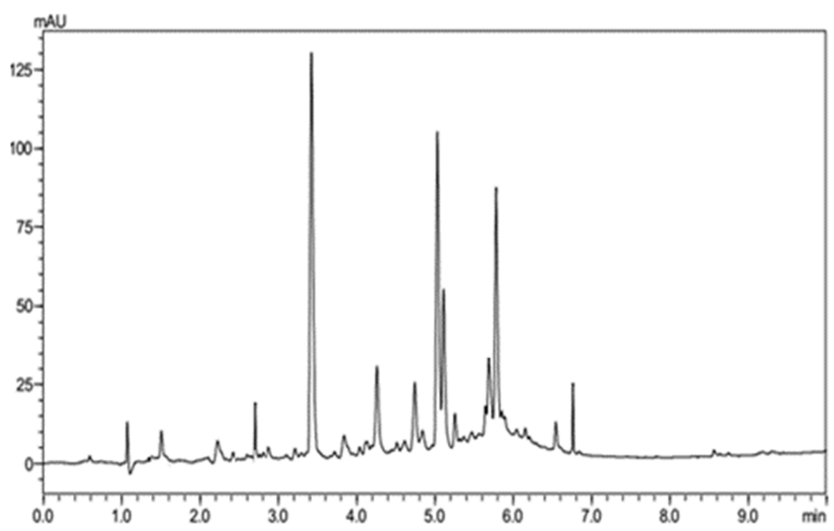

Figure 3. Mesquitol standard HPLC chromatogram.

\subsubsection{Precision}

The developed methods precision, was assessed in terms of its repeatability and intermediate precision both intraday and inter-day. The precision, was expressed in terms of percentage relative standard deviation (\% RSD) and it was determined by analysing the mesquitol solution over the ranges of between 10-160 three times a day over a period of three days.

The $\%$ RSD ranged from $0.0046 \%$ to $0.1095 \%$ signifying that the developed method was highly reproducible thus indicating that the variation of analysis would not be significant between different concentrations whether low or high.

\subsubsection{Accuracy}

The methods accuracy, was established by performing recovery experiments with the results ranging from $98.211 \%$ to $99.642 \%$ as in table 3 below. The percentage recovery was within (98\%-100\%) hence the method was deemed to be accurate.

Table 3. Recovery results for intraday and interday analysis of mesquitol standards.

\begin{tabular}{lllllll}
\hline Conc. $\boldsymbol{\mu g m L}^{-1}$ & Day 1 & Day 2 & Day 3 & Mean & S. D & RSD \\
\hline 0 & 99.301 & 99.242 & 99.642 & 99.395 & 0.21593 & 0.002172 \\
20 & 99.465 & 99.316 & 99.214 & 99.337 & 0.12623 & 0.001271 \\
40 & 98.324 & 98.211 & 98.109 & 98.215 & 0.10755 & 0.001095 \\
80 & 98.713 & 99.213 & 99.435 & 99.120 & 0.36981 & 0.003731 \\
160 & 99.514 & 98.937 & 99.147 & 99.199 & 0.29204 & 0.002944 \\
Mean & 99.0634 & 98.9838 & 99.1094 & & & \\
S. D & 0.52208 & 0.455238 & 0.592206 & & & \\
RSD & 0.00527 & 0.004600 & 0.005975 & & & \\
RSD $\%$ & 0.52701 & 0.46001 & 0.597552 & & & \\
\hline
\end{tabular}

\subsubsection{Limit of Detection and Quantification.}

The two were calculated by the simultaneous analysis of mesquitol using the obtained standard deviations of the already determined analytical recoveries and were found to be $9.18 \mu \mathrm{g} / 1$ and $27.84 \mu \mathrm{g} / 1$ respectively. The LOD, was approximately three times lower than the LOQ hence satisfying the ICH recommendations on the relationship between the two [14]

\subsubsection{Robustness}

The methods robustness was assessed by the deliberate changing of chromatographic conditions only one at a time. The flow rate was first varied from $7.5 \mathrm{~mL} \mathrm{~min}^{-1}$ to $0.1 \mathrm{~mL}$ $\mathrm{min}^{-1}$. The pump pressures of the HPLC, were then equally varied from 8.0 $\mathrm{MPa}$ to a maximum of $15.0 \mathrm{MPa}$. The deliberate variations of the two, showed that the method remained unaffected by the small changes of the two variables 
showing the methods robustness.

\subsubsection{Selectivity}

The methods selectivity was confirmed from the observed chromatograms with a comparison to the one for pure mesquitol. The retentions time of the observed chromatograms of pure mesquitol and our samples were the same confirming the methods specificity.

\subsubsection{Linearity}

The linearity of the method, was established within the ranges $10 \mu \mathrm{g}-160 \mu \mathrm{g} / \mathrm{l}$ with triplicates of the concentrations $10,20,40,80$ and $160 \mu \mathrm{g} / \mathrm{l}$. this resulted into the regression equation of a straight line of the form $y=20885 x+7980.6$. The correlation coefficient $\left(\mathrm{R}^{2}\right)$ was 0.9983 showing a good correlation between the sample concentrations and the peak response areas as shown in figure 4.

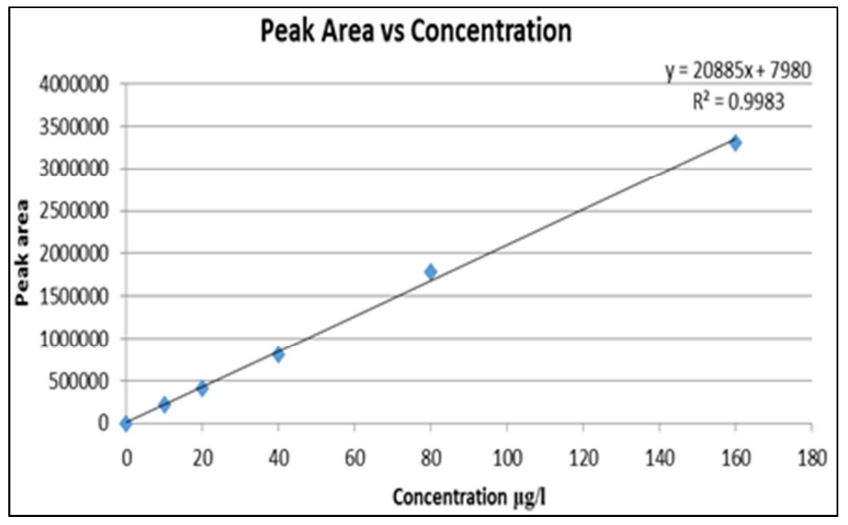

Figure 4. Calibration curve for mesquitol.

\subsection{Quantification of Mesquitol in P. juliflora Crude Extracts}

The developed and validated HPLC method was applied in determination of the Mesquitol content in the crude extracts and tabulated appropriately as follows in table 4 .

Table 4. Mesquitol abundances in crude extracts.

\begin{tabular}{lll}
\hline Sample & $\begin{array}{l}\text { Mesquitol abundances in } \\
\text { crude extracts }(\boldsymbol{\mu g} / \mathbf{m L})\end{array}$ & \% abundance \\
\hline WAb-Ace & 634.162 & 4.943 \\
& 642.893 & 5.001 \\
& 615.399 & 4.796 \\
WB1-Ace & 209.094 & 1.630 \\
& 211.255 & 1.647 \\
& 203.659 & 1.587 \\
DBl-Ace & 189.367 & 1.473 \\
& 181.245 & 1.413 \\
& 190.026 & 1.481 \\
DAb-Ace & 533.412 & 4.158 \\
& 542.198 & 4.226 \\
& 561.426 & 4.376 \\
\hline
\end{tabular}

KEY: WBl-Ace: acetonic extracts of plant below 4 years of harvested in the wet season.

WAb-Ace: acetonic extracts of plant above 4 years harvested in the wet season. DBl-Ace: acetonic extracts of plant below 4 years harvested in the dry season. Dab-Ace: acetonic extracts of plant above 4 years harvested in the dry season.

\subsection{Seasonal Variations}

In the tropics the main determinants of seasonal variations are usually rainfall, temperature and relative humidity. These have been realized to have impact on phyto-depositions of secondary metabolites in plants [16]. Information on rainfall, temperature and relative humidity trends is important especially in the Savannah where these climatic variables are becoming increasingly unpredictable. The average monthly rainfall trend in Baringo County geographical areas are presented in figure 5 .

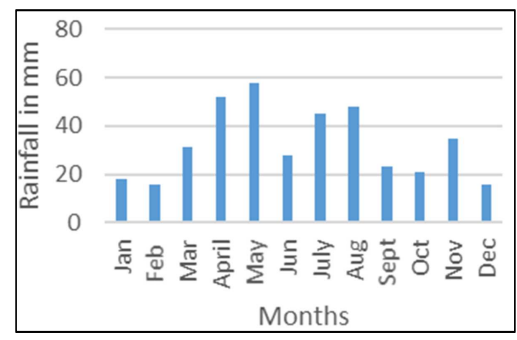

Figure 5. The average monthly rainfall trend in Baringo County.

Marigat sub county is a semi-arid region of about $900 \mathrm{~m}$ above sea level with an average rainfall of about $500 \mathrm{~mm}$ per annum. The rains are $30 \%$ reliable with the wet season being bimodal with the long rains being experienced in the months of late March to early July and short rains in the months of October and early November. The dry season is between the months of late November to early March [17].

The wet seasons are characterised by significant rainfall hence availability of water, high humidity and moderate temperatures of around $25^{\circ} \mathrm{C}$ with the dry seasons being characterized by little or no rainfalls, low humidity and temperature highs of up to $35^{\circ} \mathrm{C}$

\subsection{Mesquitol Abundance with Seasonal Aspects Variations}

The results suggested that, the amount of mesquitol content varied as a function of the seasons during which plants were harvested with the wet season having higher mesquitol content than those harvested during the dry seasons.

It was also observed that there is a good correlation of low temperatures and good sunlight conditions as a balance of optimum conditions that lead to the accumulation of flavonoids [18].

\section{Conclusion}

A simple, accurate, specific and robust HPLC method for the quantitative evaluation of mesquitol was developed and validated according to ICH standards. Mesquitol was successfully extracted and isolated from the heartwood of the plants crude acetonic extracts.

The results showed that mesquitol does indeed greatly vary with the season of harvest, with the wet seasons having significantly higher yields than the dry seasons. The results also showed that, mesquitol abundance varies with the age of the plant, with plants over the age of 4 years having a 
significant higher amount of mesquitol than those of below the age of 4 years. Hence it can be concluded that it is best to harvest mesquitol during the wet season especially to plants that are of 4 years and above.

\section{References}

[1] Chang, S., Cheng S., Wang S. (2001). Antitermitic activity of essential oils and components from Taiwania (Taiwania cryptomerioides). Journal of Chemical Ecology, 27 (4), 717724.

[2] Rao, R. J., Tiwari, A. K., Kumar, U. S., Reddy, S. V, Ali, A. Z., Rao, J. M., (2003). Novel 3-O-Acyl Mesquitol Analogues as Free-Radical Scavengers and Enzyme Inhibitors: Synthesis biological Evaluation and Structure-Activity Relationship, Science direct: Bioorganic \& Medicinal Chemistry Letters 13 (2003) 2777-2780.

[3] Liu S, Huang H. 2014. Assessments of antioxidant effect of black tea extract and its rationals by erythrocyte haemolysis assay, plasma oxidation assay and cellular antioxidant activity (CAA) assay. J Funct Foods. 18:1095-1105.

[4] Lee, J. H., Lee, J. Y., Kim, K. N. et al. 2003. Quantitative analysis of two major flavonoid aglycones in acid hydrolyzed samples of Angelica keiskei by HPLC. - Food Sci. Biotech. 12: 415-418.

[5] Azam, M. M., Tewari J. C., Singh Y., Roy M. M. (2011). Prosopis juliflora A rich Source of Antioxidant Product. Central Arid Zone Research.

[6] Nieva-Echevarria B, Manzanos MJ, Goicoechea E, Guillen MD. 2015. 2, 6-Ditert-butyl-hydroxytoluene and its metabolites in foods. Comp Rev Food Sci Food Safety. 14:6780 .

[7] Suresh, G., Tiwari, A. K., Radha, K. M, Kumar, D. A., Prasad, R. K., Ali, A. Z., Rao, R. R., (2012). New advanced glycation end-product inhibitors from Dichrostachys cinerea. Journal of Natural Medicines, 66:213-216 ISSN 1340-3443.

[8] Vagiri M, Conner S, Stewart D, Andersson SC, Verrall S, Johansson E, Rumpunen K. 2015. Phenolic compounds in blackcurrant (Ribes nigrum L.) leaves relative to leaf position and harvest date. Food Chem. 172:135-142.

[9] Gouvea, D. R., Neto. L. G., Sakamoto, H. T., Lopes, P. N.,
Lopes, C. L. (2012) Seasonal variation of the major secondary metabolites present in the extract of eremanthus mattogrossensis less (asteraceae: vernonieae) leaves. Quim. Nova, Vol. 35, No. 11, 2139-2145.

[10] Koleva II, Van Beek TA, Linssen JPH, De Groot A, Evstatieva L. N. (2002) Screening of plant extracts for antioxidant activity: a comparative study on three testing methods. Phytochem Anal; 13:8-17.

[11] Soni, U., Braar S., Guattam, V. (2015). Effects of seasonal variations on secondary metabolites of medicinal plants. International journal of pharmaceutical sciences and research, Vol 6 (9):3654.

[12] Nascimento, F., Faqueti, A., Wilhem, J. Wittkowski, C. Tomczak, F., Borges, S., Yunes, R., Franchi Jnr, G., Nowil, A., Filho, V., Machado, M., Freitaes, R. Maheiros., A (2014) Seasonal influence and cytotocicty of extracts, fractions and major compounds from Allamanda scatti, Brazilian journal of pharmacognosy 24:545-552.

[13] Pendergrass, J. S., (1984). Non-carbohydrate organic compounds in mesquite heartwood. Master of Science Thesis, Texas Tech University.

[14] ICH Harmonised Tripartite Guideline: Stability testing of New drug substances and products available 2003 Q1A (R2). Available: http://www.ich.org/fileadmin/Public Web Site/ICH Products/ Guidelines/Quality/Q1A_R2/Step4 /Q1A_R2_Guideline.pdf (Accessed September 2011).

[15] Hossain, M. A., Khulood, A. S. R., Zawan, H. M., Afaf, M. W. Qasim, A. R. (2013). Study of total phenol. Flavonoid contents and phytochemical screening of avroius leaves extract of locally grown Thymus vulgaris. Asian Pac journ Trop biomed.

[16] Sampaio B. L., Edrada-Ebel R. and Da Costa F. B. (2016). Effect of the environment on the secondary metabolic pro file of Tithonia diversifolia: a model for environmental metabolomics of plants Science Reports 6: 29265. doi:10.1038/srep29265.

[17] Lelenguyah, G., Kabachi, S., Biwott, J. (2016). Pastoral ists' perception on trends of various climatic, social and environmental variables in Baringo county, Kenya. Journal of ecological anthropology, Vol 18, Issue No. 1.

[18] Haribal, M. and Renwick, J. A. A. 2001. Seasonal and population variation in flavonoid and alliarinoside content of Alliaria petiolata. J. Chem. Ecol. 27: 1585-1594. 